\section{DISEÑO INSTRUCCIONAL DE LA EDUCACIÓN EN LÍNEA \\ USANDO EL MODELO ASSURE}

INSTRUCTIONAL DESIGN OF ON LINE COURSES

USING THE ASSURE MODEL

Alirio Antonio Dávila ${ }^{1}$

Judith Francisco Pérez **

Universidad Centroccidental Lisandro Alvarado

Recibido: 09-04-07

\section{RESUMEN}

Este trabajo tiene como propósito divulgar una experiencia educativa de los autores en la Universidad Centroccidental Lisandro Alvarado (VCLA) sobre la adaptación y aplicación del Modelo ASSURE (Heinich, Molenda, Russell, y Smaldino, 2002) para guiar el diseño instruccional de cursos en línea. Se comprobó que el modelo ASSURE es aplicable y pertinente en un contexto de formación a distancia y no demanda necesariamente la intervención de equipos multidisciplinarios de planificadores. E seguimiento adecuado del modelo permite que los planificadores del proceso instruccional en línea dispongan de un plan de acción detallado y bien definido para el análisis, diseño, preparación y evaluación de la instrucción. E modelo es fácil de aplicar y facilita condiciones para llevar a cabo un proceso instruccional a distancia efectivo para el logro de aprendizajes significativos.

Descriptores: Diseño Instruccional, Modelo Assure, Educación en Línea.
Aceptado: 29-11-07

\section{ABSTRACT}

The purpose of this article is to show the authors' educational experience in the Universidad Centroccidental Lisandro Universidad Centroccidental Lisandro
Alvarado related to the adaptability and usage of the Assure Model (Heinich, Molenda, Russell, and Smaldino, 2002) to guide the instructional design of online courses. It was proved that the Assure Model is applicable and pertinent to online educational context, and furthermore it does not require the participation of multidisciplinary teams of instructional designers. An adequate use ofthe model allows the main actors ofthe online education to have a welI defined plan to analyse, design, implement, and evaluate the instructional process. The model is easy to be used and gives some conditions to facilitate an effective distance instructional process to promote the achievement of meaningful learning by the participants.

Keywords: Instructional Design, Assure Model, Online Education

${ }_{1}^{1}$ Profesor Titular, Jubilado, Universidad Centroccidental Lisandro Alvarado (UCLA). Doctor en Tecnología Instruccional y Educación a Distancia. Profesor de Matemáticas. Coordinador General del Sistema de Educación a Instruccion y Educación a Distancia. Pr

** Profesora Agregada, Docente del Decanato de Ciencias de la Salud, Universidad Centroccidental Lisandro Alvarado (UCLA). Doctora en Tecnología Instruccional y Educación a Distancia. Master en Planificación Curricular. Licenciada en Enfermería .Directora del Programa de Enfermería, Decanato de Ciencias de la Salud

En materia de planificación de la instrucción, todo docente debe ser metódicamente organizado. Esta premisa se convierte en un requisito imprescindible para el ejercicio de la docencia a distancia, dada la separación física entre facilitadores y aprendices. En este sentido, el planeamiento de acciones para ejecutar con calidad un proceso instruccional presencial o a distancia requiere fundamentalmente que el profesor esté preparado y motivado para diseñar la instrucción con buen sentido pedagógico y eficacia.

En la literatura sobre este tema, diversos autores han presentado elementos suficientes para señalar que la motivación de los aprendices, y el logro de los resultados de aprendizaje debidamente intencionados o propuestos, están asociados en buena medida con el diseño instruccional que se ponga en práctica (Dick, Carey y Carey, 2001; Gustafson y Branch, 2002; Cookson, 2003). Por ejemplo, según Cookson, el diseño instruccional provee orientación, consejos, y soporte tanto para el diseñador como para el instructor y los participantes; y, por ser un proceso cíclico, posibilita el mejoramiento continuo de la instrucción.

Ahora bien, para orientar la planificación instruccional de los docentes, se conocen diversos modelos; entre ellos: Modelo de Ely y Gerlach; Modelo ASSURE de Heinich, Molenda, Russell y Smaldino; Modelo de Newby, Stepich, Lehman, y Russell; Modelo de Morrison, Ross y Kemp. Estos modelos fueron examinados por Gustafson y Branch (2002).

Los autores de este artículo seleccionaron y evaluaron el Modelo ASSURE como un marco conceptual y comunicacional apropiado para guiar, dirigir, administrar y evaluar procesos instruccionales en línea de alta calidad académica. La aplicación del modelo no requiere una alta experticia de los docentes en teorías de diseño instruccional. Tampoco se requiere de inversiones elevadas en recursos administrativos o tecnológicos.

Este planteamiento de planificación instruccional sustentado en el Modelo ASSURE, se basó en la experiencia acumulada por los autores en su participación y administración de cursos en línea en la Universidad Centroccidental Lisandro Alvarado (UCLA), Barquisimeto, Venezuela. Particularmente, se aplicó sobre la plataforma MOODLE (http://moodle.org)

\section{IMPORTANCIA DE LA PLANIFICACIÓN INSTRUCCIONAL}

Según Gagné y Briggs (2001), la razón fundamental para planificar la enseñanza es hacer posible la consecución de un cierto conjunto de objetivos de aprendizaje 
propuestos. Es por ello que existe una relación íntima entre el conocimiento construido, la manera en la cual el alumno aprende, y el saber qué hacer para ayudarlo a aprender mejor (Ausubel, Novak y Hanesian, 2000).

En este sentido, la enseñanza es el encauzamiento deliberado de los procesos de aprendizaje a través de los lineamientos y estrategias empleadas por el facilitador. Esto se explica así porque mientras el aprendizaje tiene carácter "individual y endógeno" (Díaz-Barriga y Hernández, 2002: 3) y se activa mediante procesos internos inherentes a quien aprende, la enseñanza, es "la ayuda para que otras personas aprendan” (Gagné y Briggs, 2001: 13). Naturalmente, esa ayuda debe ser responsabilidad especial del facilitador, quien la ofrecerá de la mejor manera posible; es decir, fomentando situaciones de aprendizaje que coloquen al estudiante como responsable directo de su propia formación y crecimiento intelectual.

Es por ello que en la educación formal, aprendizaje y enseñanza ocurren simultáneamente y su planificación y regulación exige la revisión de un marco teórico o modelo que oriente la organización de todas las variables involucradas en el proceso de instrucción. Es en este punto donde cobran relevancia los modelos de diseño instruccional.

\section{Modelos de Diseño Instruccional}

De acuerdo con Schlosser y Simonson (2002), un modelo de diseño instruccional (MDI) representa un marco referencial o proceso sistemático para desarrollar instrucción de manera directa o mediada. Esto incluye una planificación del curso en cuanto a su fundamentación, competencias a lograr, objetivos de aprendizaje; selección de medios, métodos, estrategias de enseñanza y de aprendizaje; así como, técnicas e instrumentos de evaluación. Incluye además, organizar la realimentación que requerirán estudiantes y facilitadores para determinar en que medida los objetivos fueron logrados y que tan bien funcionaron las estrategias de enseñanza y aprendizaje.

Adicionalmente, para Gustafson y Branch (2002), los MDI ayudan a conceptualizar representaciones de las realidades del desarrollo de un proceso de enseñanza y aprendizaje, simbolizadas por las actividades y recursos que se ponen en marcha en búsqueda de una instrucción de calidad medida por el logro efectivo de los objetivos propuestos. Así, se caracterizan por promover esencialmente cinco actividades: (a) análisis de las necesidades y características de los aprendices, (b) diseño de un conjunto de especificaciones para un efectivo, eficiente y relevante ambiente o escenario para el aprendizaje, (c) desarrollo de todos los materiales y actividades para el aprendizaje, (d) implementación de la instrucción resultante y (e) evaluaciones sumativas y formativas de los resultados del desarrollo instruccional. Estas cinco actividades son referidas frecuentemente como ADDIE y representan un modelo genérico de diseño instruccional.

De esta manera, el modelo ADDIE constituye una conceptualización genérica de planificación instruccional que consiste de cinco elementos: Análisis, Diseño, Desarrollo, Implementación y Evaluación. En general, la tarea esencial de un buen educador para buscar la excelencia y calidad de su rol como instructor consiste en practicar y poner en marcha de la mejor manera posible estos cinco elementos genéricos de diseño instruccional.

\section{Supuestos y Ventajas de los MDI}

Según Gustafson y Branch (2002), los MDI proveen herramientas conceptuales y comunicacionales que pueden ser usadas para visualizar, dirigir y administrar procesos directos o mediados para la creación y entrega de instrucción con alto nivel de calidad. En el sondeo sobre un significativo número de ellos, Gustafson y Branch identificaron los supuestos presentados por los creadores de los MDI examinados; entre éstos enfatizaron los siguientes:

1. Sirven como marcos conceptuales, que controlan y facilitan herramientas comunicacionales para analizar, diseñar, crear y evaluar procesos de aprendizaje guiado, concernientes con amplios ambientes educacionales o con reducidas acciones educativas de entrenamientos

2. Cuanto mayor sea la compatibilidad entre los MDI y sus orígenes teóricos, filosóficos, y contextuales, en esa misma medida aumentará el potencial para lograr el éxito del MDI en la construcción de ambientes efectivos de aprendizaje. Es decir, si un MDI se sustenta en supuestos constructivistas del aprendizaje, entonces su aplicación debe estar firmemente orientada por tales supuestos y concepciones teóricas.

3. Ayudan a tomar en cuenta los múltiples escenarios de los estudiantes en cuanto a sus experticias como aprendices, las múltiples interacciones que pueden ocurrir durante el proceso de aprendizaje y la variedad de contextos en los cuales el aprendizaje está situado.

4. Ninguno es completamente adecuado para satisfacer los variados diseños y ambientes de desarrollo en los cuales trabajan los especialistas en planificación instruccional. Por lo tanto, estos especialistas deben ser competentes en aplicar (y posiblemente adaptar) una variedad de modelos para satisfacer los requerimientos de situaciones específicas. 
5. El interés sobre los MDI continuará, aunque el nivel de aplicación variará en correspondencia con cada contexto o situación.

Por su parte, Smith y Ragan (1999), señalaron algunas ventajas que se derivan del diseño sistemático de la instrucción, a través de los MDI:

1. Estimulan una concentración de acciones en favor de los aprendices. En un alto grado, los aprendices son el centro de la instrucción.

2. Soportan una eficiente, efectiva y atractiva instrucción. La consideración prioritaria de los aprendices y el diseño de estrategias apropiadas promocionan una instrucción interesante. El proceso de evaluación formativa ofrece oportunidades para revisar la instrucción y hacerla más efectiva, eficiente e interesante.

3. Favorecen la coordinación entre diseñadores, desarrolladores y aquellos que implementan la instrucción. El proceso sistemático de planificación y la resultante documentación escrita permite la comunicación y coordinación entre los individuos involucrados en el diseño, producción y entrega de la instrucción.

4. Facilitan la difusión, diseminación y adopción de productos. Debido a que los resultados de un diseño instruccional sistemático son en realidad "productos" físicos que pueden ser duplicados, distribuidos y usados como referentes de buena práctica educativa para el logro de ambientes efectivos de aprendizaje.

5. Soportan el desarrollo de sistemas o medios alternativos para la entrega de instrucción. Muchos de los resultados de un proyecto de diseño instruccional son independientes de la forma específica que toman para materializarse como productos finales (documentos impresos, videos, imágenes, etc.). Subsiguientes proyectos pueden tomar esos productos como referentes para desarrollar otros medios alternativos de entrega de la instrucción. Si la entrega de instrucción mediante un video funcionó bien para una determinada situación, es posible que para otra situación se requiera una entrega de la misma instrucción pero con otro medio.

6. Facilitan la congruencia entre los objetivos, las actividades y la evaluación. El enfoque sistemático de un diseño instruccional ayuda a asegurar que lo que es enseñado es lo que necesitan los aprendices para alcanzar los objetivos de aprendizaje propuestos y que la evaluación será precisa y apropiada para medir esos logros.

7. Representan un marco apropiado para resolver problemas de aprendizaje. La planificación sistemática de la instrucción y su diseño conllevan a los instructores, diseñadores y desarrolladores a tomar en cuenta múltiples variables que inciden en determinados problemas de aprendizaje, y esto hace que la planificación sea un proceso dinámico para la búsqueda de soluciones efectivas.

En este orden de ideas, uno de los MDI más sencillos y ampliamente utilizados es el modelo ASSURE. Propuesto por Heinich et al. (2002), este modelo es útil para guiar y asegurar la planificación sistemática paso a paso, del más conocido evento instruccional: la lección. Según la taxonomía de modelos de diseño instruccional propuesta por Gustafson y Branch (2002), el Modelo ASSURE se orienta hacia la planificación instruccional de los procesos de enseñanza y aprendizaje que se dan en un aula convencional. Sus creadores advirtieron que se trataba de un modelo menos ambicioso y de menor alcance que aquellos modelos de planificación instruccional concebidos para guiar procesos representados por sistemas instruccionales mucho más complejos, tales como cursos o programas académicos completos.

Con el acrónimo ASSURE se abrevian 6 fases:

A - analizar a los aprendices (Analyze learners),

S - formular los objetivos de aprendizaje (State learning objectives),

S - seleccionar métodos, medios y materiales (Select methods, media and materials),

U - utilizar métodos, medios y materiales (Utilize methods, media and material),

$\mathrm{R}$ - requerir la participación de los aprendices (Require learner participation), y

E - evaluar (Evaluate and revise).

\section{DESCRIPCIÓN DE LA EXPERIENCIA DE INSTRUCCIÓN EN LÍNEA USANDO EL MODELO ASSURE}

Análisis de los aprendices. En esta fase identifican las capacidades, necesidades e intereses de la audiencia. Se resalta la importancia de conocer las características generales y específicas de los participantes, tales como: edad promedio del grupo, nivel socio-económico y cultural, habilidades o competencias de entrada, experticia en el uso de determinada tecnología, actitudes hacia el tema de la lección planteada, estilos de aprendizaje.

Algunas preguntas que ayudaron a los autores en la construcción del instrumento aplicado en 
esta fase del Modelo ASSURE fueron las siguientes:

¿Se tiene información sobre el nivel de instrucción previo de los aprendices?

¿Están los aprendices suficientemente interesados y motivados para seguir el curso?

¿Se ha recogido información sobre los conocimientos de entrada y conocimientos previos de los aprendices con relación al tema de estudio?

¿Se ha tomado alguna decisión con respecto a los estilos de aprendizaje de los aprendices en situaciones de formación en línea?

¿Será necesario organizar algunos encuentros presenciales o toda la instrucción se puede facilitar en línea?

¿Se ha recogido información sobre la experticia de los aprendices en el uso de correo electrónico, Internet, y determinado software o tecnología?

¿Se tiene información sobre el rechazo o aceptación de los aprendices para la formación en línea?

¿Se tiene información precisa sobre las posibilidades de acceso de los aprendices a la instrucción entregada vía Internet?

En función de las respuestas obtenidas, los autores diseñaron la siguiente prueba exploratoria con la finalidad de analizar la audiencia:

\section{Prueba Exploratoria}

\section{Instrucciones:}

Estimado participante, la siguiente prueba se ha diseñado para identificar algunas características generales y particulares de la audiencia del presente curso. Sus respuestas serán empleadas para planificar y orientar el desarrollo de la instrucción; por lo tanto, requerimos complete la información solicitada. Por favor, no deje datos sin completar. En algunos casos, bastará marcar una X como respuesta; mientras que en otras, se requerirá que escriba una respuesta corta de cinco líneas como máximo.

Su nombre y apellido:
Teléfonos: Oficina: Móvil:

Conocimientos Previos

1. ¿Conoce usted la plataforma Moodle? Si __. No. afirmativa, ¿En qué rol conoce usted la plataforma Moodle? Si su respuesta fue Profesor Administrador

2. En general, su conocimiento de Moodle es:
a. Nulo: Bajo
Moderado Suficiente

3. Cómo califica usted su manejo del sistema Windows para operar un computador:

a. Insuficiente Suficiente: Excelente:

4. ¿Posee usted alguna cuenta de correo electrónico? Si No

5. Cómo califica usted su manejo del servicio de correo electrónico:

a. Insuficiente Suficiente: Excelente:

6. Cómo califica usted su experticia en el uso de Internet:

a. Insuficiente Suficiente: Excelente:

7. ¿ ¿ Tiene usted facilidades de acceso a Internet desde su domicilio? Si __ NO

8. Cómo califica usted sus conocimientos acerca de la modalidad de educación a distancia: a. Insuficiente Suficiente: Excelente:

Factores de Motivación

1. ¿Por qué se ha interesado usted en asistir a este curso? Por favor, explique.

2. ¿ ¿ ¿ se siente usted cómodo con el uso de tecnología digital en el desempeño de su labor docente? Si No: No sabe: 
3. ¿El uso de Internet como medio para la entrega de instrucción le produce a usted alguna motivación intrínseca? Si . No: No Sabe: Por favor, explique.

4. ¿Cómo percibe su estado interno motivacional para atender este curso?

a. Bajo Moderado: Alto:

5. ¿Cuántas horas diarias estima usted que dedicará al estudio y seguimiento de este curso? 1 ó menos . Entre 1 y 2: Más de 2:

6. ¿Cómo preferiría usted el desarrollo de este curso?

a. Presencial __. Semipresencial: __. A distancia:

Una vez tabulada la prueba, los resultados determinaron las siguientes características generales de los participantes: (a) $60 \%$ prefería que el curso se ofreciera totalmente a distancia y el resto, 40\%, sugería la realización de al menos un encuentro presencial; (b) $80 \%$ tenía facilidades de acceso a Internet desde sus domicilios y experticia básica en su manejo, el resto accedía desde la misma universidad; (c) todos disponían de cuentas privadas de correo electrónico y experticia en el envío y descarga de archivos adjuntos; (d) ninguno tenía experiencia previa en el uso de la plataforma Moodle; (d) algunos usaban la plataforma SABER para administrar cursos en línea en la UCLA; (e) todos tenían una actitud favorable para usar Moodle y ofrecer cursos en líneas al finalizar el curso.

Con base en la información proporcionada por los participantes, los facilitadores concluyeron que se debía ofrecer un curso básico o introductorio. Se decidió ofrecer el curso en modalidad mixta, con dos encuentros presenciales, inicio y fin de curso, más entrega de instrucción vía Internet.

Formulación de los objetivos de aprendizaje. En esta fase se precisa en términos de conductas observables y medibles lo que se espera que cada aprendiz adquiera y domine al final de cada experiencia de aprendizaje. En general, se pretende determinar cuáles serán sus conocimientos (saber conocer), actitudes y valores (saber ser) y desempeños (saber hacer), a lograr al culminar la experiencia de aprendizaje. Básicamente, la formulación precisa y correcta de los objetivos de aprendizaje debería evitar que la instrucción y la evaluación vayan por sentidos opuestos. Cuando los objetivos son formulados en términos de desempeño observable, representan una guía para clarificar la definición de actividades de aprendizaje en función de su logro (Gronlund, 2000).
Heinich et al. (2002) propusieron un esquema fácil de aplicar para la formulación de objetivos de aprendizaje, el formato ABCD, con esta significación:

A - Audiencia (Audience). ¿Quiénes son los aprendices?;

B - Conducta (Behavior). ¿Cuál será la conducta que demostrarán los aprendices?;

C - Condiciones (Conditions). ¿En cuáles condiciones la conducta será observada?;

D - Criterio (Degree or Criteria). ¿Cuál será el criterio que satisfaga el nivel de logro de la conducta adquirida?

Algunas de las preguntas que orientaron a los autores en esta fase del modelo fueron:

¿Están los objetivos formulados en términos de lo que los aprendices deben saber conocer, saber hacer o saber ser?

¿Se han formulado objetivos que estimulen altos procesos cognitivos?

¿Son específicos los resultados de aprendizaje previstos en los objetivos?

¿Se han formulado los objetivos con el uso de verbos que señalan inequívocamente conductas observables y medibles?

¿Los objetivos están formulados de manera que ayuden al estudiante a pasar progresiva y crecientemente por los diferentes niveles cognitivos, actitudinales y psicomotrices? Además, ¿esa organización dispone al estudiante a realizar esfuerzos intelectuales bajos, intermedios y altos?

En el caso de la presente experiencia el programa instruccional estuvo planificado con base en el enfoque de competencias; por lo cual, los autores se aseguraron que los objetivos de aprendizaje dieran cuenta de los saberes necesarios para promover en el estudiante el logro de esas competencias. Por ello, fue necesario precisar respuestas a las siguientes preguntas:

¿Cuál sería la competencia general a lograrse por los aprendices?

¿Cuáles serían las competencias específicas asociadas con el logro de la competencia general?

¿Cuáles deberían ser las evidencias de desempeño (saber hacer), de conocimiento 
(saber conocer) y actitudinales (saber ser) necesarias para el logro de esas competencias?

Planteado de esta manera, los saberes serán coherentes y permitirán el logro de las competencias establecidas, quedando a juicio del facilitador si plantea objetivos específicos de aprendizaje o no lo hace (Tobón, 2007)

En tal sentido, para el curso básico de plataforma Moodle se realizaron las siguientes formulaciones: Competencia General - Utiliza en forma autónoma e innovadora la plataforma Moodle para la creación y gestión de cursos en línea. Competencias Específicas: a) Crea un curso en línea utilizando la plataforma Moodle; y b) Gestiona el uso de los componentes básicos de Moodle para la entrega de instrucción en línea.

Con relación a la competencia específica, crea un curso en línea utilizando la plataforma Moodle, se determinan las siguientes evidencias de desempeño, de conocimiento y de actitudes y valores:

1. Evidencias de conocimiento (saber conocer): Diferencia las categorías del Portal Institución-Moodle, diferencia entre cursos disponibles o no disponibles para los estudiantes; compara los diferentes recursos y actividades que ofrece la plataforma Moodle, diferencia las etapas de planificación de un curso en línea, diferencia recursos y actividades instruccionales y de aprendizaje.

2. Evidencias de desempeño (saber hacer): Planifica el desarrollo del curso en formato de semanas o temas incluyendo recursos y actividades pertinentes para la instrucción, configura el curso en línea en diferentes formatos, publica su curso en la categoría pertinente a su programa académico, activa y desactiva modo de edición de un curso propuesto; edita información de un curso propuesto; crea grupos de trabajo (secciones) diferentes; solicita informes sobre el desempeño de los participantes.

3. Actitudes y Valores (saber ser): Consulta a su facilitador de manera directa, respetuosa y amigablemente; cumple puntualmente con las asignaciones propuestas; participa en las actividades programadas; tolera y muestra respeto por las opiniones que le son adversas en los encuentros asincrónicos o sincrónicos programados.

Con relación a la competencia específica, gestiona el uso de los componentes básicos de Moodle para la entrega de instrucción en línea, se determinaron las siguientes evidencias de desempeño, de conocimiento y de actitudes y valores:

1. Evidencias de desempeño (saber hacer): Presenta un mensaje de bienvenida y motivador del curso; publica mensajes con orientaciones semanales para el estudio indepen- diente de un determinado tema; publica actividades generadoras de debate para sesiones de Chat y foros de discusión en concordancia con los objetivos a lograr en un determinado tema; propone lecturas analíticas de artículos o materiales publicados en Internet; publica materiales didácticos para promover el aprendizaje independiente; planifica actividades que requieran la estrategia de trabajo cooperativo; publica autoevaluaciones formativas.

2. Evidencias de conocimiento (saber o conocer): Diferencia las estrategias virtuales de enseñanza y aprendizaje - foros de discusión, Chat, y trabajo cooperativo; identifica las condiciones para favorecer el aprendizaje independiente; reconoce las condiciones requeridas para la mediación del aprendizaje independiente.

3. Actitudes y Valores (saber ser): Consulta a su facilitador de manera directa, respetuosa y amigable; cumple puntualmente con las asignaciones propuestas; participa en las actividades programadas; tolera y muestra respeto por las opiniones que le son adversas en los encuentros sincrónicos y asincrónicos programados.

Seleccionar Métodos, Medios y Materiales. En esta fase, el facilitador determina las ayudas didácticas o recursos disponibles para el desarrollo de la instrucción en función del logro de los objetivos a lograr. Escoge entre métodos, medios y materiales disponibles, modifica los existentes o diseña unos nuevos, esenciales para el logro de los objetivos. Si una estrategia de enseñanza funciona bien en modalidad presencial, probablemente requiera algunos ajustes para que funcionen bien a distancia. En cualquier caso, debe asegurarse que los materiales de apoyo estén disponibles al inicio del curso y no producirlos sobre la marcha.

Como regla general, se debe centrar la planificación instruccional en la adopción de métodos y estrategias que demanden actividades a ser realizadas por los participantes y no por el profesor. Se toma en cuenta que según los supuestos del enfoque constructivista del aprendizaje, los estudiantes construyen activamente nuevos conocimientos a medida que interactúan con su entorno (Driscoll, 2000).

Entre otras, algunas preguntas que guiaron a los autores en esta fase de la planificación instruccional fueron las siguientes:

¿Qué tipo de materiales de apoyo pueden emplearse para facilitar diversas situaciones de aprendizaje que demandan la participación activa, reflexiva y analítica de los aprendices?

¿Qué tipo de experiencias pueden emplearse para facilitar la comprensión global de los temas estudiados?

¿Se podría considerar la solicitud de trabajos escritos? ¿Con qué propósito? 
¿Existen problemas, proyectos o estudios de casos a ser propuestos?

Si van a utilizarse materiales previamente concebidos para la educación presencial, ¿`se han ajustado a la modalidad de educación a distancia?

¿Se ha probado con suficiente anticipación el funcionamiento y efectividad de algún método, medio o nueva tecnología?

¿Se ha comprobado si todos los participantes tienen acceso a Internet y tienen activas sus cuentas de correo electrónico?

¿Será pertinente la apertura de foros de discusión o sesiones de Chat? ¿Qué tipo de orientación puede ofrecerse para favorecer y mantener el interés de los participantes?

¿Será apropiado proponer asignaciones en grupos de trabajo cooperativo?

¿Será necesario preparar una encuesta u otro instrumento de sondeo de opinión?

¿Se elaborarán lecciones interactivas?

¿Se organizarán actividades tipo talleres

¿Se ha preparado alguna autoevaluación del tema?

Para el caso de la presente experiencia de un curso básico sobre plataforma Moodle, las respuestas a las preguntas planteadas permitieron organizar modos de interacción a través de foros de discusión, sesiones de Chat y trabajos asignados en equipos colaborativos. Asimismo, siguiendo los principios pedagógicos que sustentan la plataforma Moodle, se propusieron situaciones de aprendizaje centradas en los participantes, las cuales favorecían que fuesen ellos mismos quienes ofrecieran explicaciones, resúmenes, o puntos de vistas sobre los temas tratados.

Específicamente, se planificó el uso de un tutorial videográfico (Hernández, 2006) para el manejo de la plataforma Moodle; se asignó un ensayo individual, referido a la concepción que tiene cada participante sobre la evaluación de los aprendizajes a distancia; se diseñó un foro de discusión sobre los principios pedagógicos que sustentan la plataforma Moodle, con énfasis en determinar la posición de cada participante con relación al enfoque constructivista del aprendizaje a distancia; se formularon preguntas activadoras de procesos del pensamiento analítico, crítico, y reflexivo; se plantearon problemas cuya resolución requiriera altos niveles cognitivos y transferencia de conocimientos; se solicitaron resúmenes que activaran procesos cognitivos de síntesis y facilitaran la concreción de puntos esenciales, principios y teorías; se elaboró una autoevaluación en línea; y se realizaron dos encuentros presenciales, uno al inicio y otro al final del curso.

Utilizar Métodos, Medios y Materiales. Esta fase se oriente el uso adecuado de los recursos disponibles. Idealmente, hacer uso adecuado de un medio, método o material, significa centrar en los aprendices el uso de los recursos para el desarrollo de sus propias actividades de aprendizaje.

Algunas de las preguntas que orientaron a los autores en esta fase del estudio fueron:

¿Los recursos o medios seleccionados están disponibles para todos los participantes?

¿Conocen todos los participantes el sitio Web del curso y sus contraseñas para accederlo?

¿Se establecieron en forma clara y explícita las reglas para las intervenciones de los participantes en foros y sesiones de Chat?

¿Se establecieron claramente las reglas para la entrega de trabajos escritos?

¿Se probó el funcionamiento adecuado de los medios o software recomendados?

¿Funcionan los enlaces o hipervínculos propuestos en los materiales?

¿Se han publicado oportunamente los materiales de estudio?

¿Se han precisado los problemas, proyectos y trabajos escritos a ser propuestos?

¿Se han verificado las respuestas correctas de las autoevaluaciones o ejercicios propuestos?

¿Se dispone de medios alternativos para la entrega de la instrucción en caso de que falle la entrega por Internet?

El análisis de las respuestas obtenidas condujo a los autores a planificar lo siguiente: se dispuso de un $\mathrm{CD}$ con las orientaciones generales y materiales para el desarrollo del curso y se previó la comunicación por vía correo electrónico en caso de fallas de la plataforma; el ensayo individual sobre factores críticos de la evaluación de los aprendizajes a distancia tendría una extensión máxima de tres páginas tamaño carta, a espacio sencillo, con márgenes simétricos de 2.54 cms; se elaboró, validó y probó una autoevaluación en línea con la finalidad de ayudar al participante a valorar sus logros de aprendizaje en el curso; y se elaboró una encuesta para verificar la calidad del curso, la cual sería aplicada durante el encuentro presencial al culminar el curso.

Requerir la Participación de los Aprendices. En esta fase se precisan las actividades y estrate- 
gias de aprendizaje centradas en los aprendices que les demanden una participación activa. Sin interacción no hay ni tiene sentido la educación a distancia (Fainholc, 1999). En un ambiente de educación en línea, la interacción centrada en los aprendices significa fundamentalmente que ellos deben leer analíticamente y escribir en forma argumentada. Según Pelz (2004), para el logro de aprendizajes significativos, leer y escribir son procesos cognitivos superiores a escuchar y hablar, como ocurre frecuentemente en un aula tradicional.

Se resalta entonces la importancia de mantener a los estudiantes involucrados activamente en sus propios procesos de aprendizaje, creando intencionalmente diversas situaciones de aprendizaje que los obligue a interactuar con los contenidos, con los facilitadores, con sus compañeros de curso y con otros expertos. También es clave incluirle en los materiales de estudio algunas preguntas de control a manera de cuestionarios sobre lo escrito, en el entendido que las preguntas se formulan para activar procesos cognitivos superiores de análisis, síntesis, evaluación, comparación, etc., y por ende son herramientas para el aprendizaje. Así, el docente asume el rol de mediador en los encuentros del aprendiz con los nuevos conocimientos y propone actividades que faciliten estos encuentros.

Algunas preguntas que orientaron a los autores en esta etapa del modelo fueron las siguientes:

¿Qué tipo de actividades pueden ser planteadas para fomentar los tipos de interacción aprendiz-facilitador, aprendiz-aprendiz, aprendiz-contenido?

¿Será realmente necesario crear en este curso, foros de discusión que generen opiniones divergentes?

¿Será necesario crear foros donde los aprendices sólo participen aportando respuestas?

¿Será conveniente crear foros donde los aprendices puedan agregar nuevos temas y ser ellos mismos potenciales moderadores de la discusión?

¿Será realmente necesario crear un foro por cada tema del curso?

¿Cómo podrían organizarse los foros de discusión o sesiones de Chat de manera que generen opiniones divergentes?

¿Podrían organizarse algunos foros donde los aprendices sean los moderadores y generadores de las preguntas o temas de debate?

¿Se propondrán trabajos individuales o en grupos cooperativos?

¿Se precisaron en forma clara y explícita los roles de los participantes en equipos de trabajo cooperativo?

¿Se han precisado tareas de investigación que requieran localizar sitos Web relacionados con los temas en estudio?

¿Qué tipo de criterios deben tomarse en cuenta para valorar las intervenciones de los participantes en los foros de discusión?

Una vez analizadas las respuestas emitidas, esta información se cotejó con los siguientes enunciados teóricos:

Uno de los propósitos fundamentales de toda plataforma tecnológica matriz de un entorno virtual de aprendizaje es facilitar herramientas para mantener atrapados activamente a los estudiantes en interacciones, sincrónicas o asincrónicas, con sus profesores y el resto de compañeros de curso. Tales plataformas soportan un conjunto de actividades centradas en los aprendices que les demandan una participación activa y protagónica. Sin interacción social no tiene sentido la educación a distancia (Fainholc, 1999), y mucho menos en los entornos virtuales para el aprendizaje en línea cuyas filosofías de gestión educativa se fundamentan en el constructivismo social como enfoque del aprendizaje. Entre esas actividades, los foros de discusión o de aprendizaje interactivo representan un medio ideal para que los alumnos participen activamente en el desarrollo de los cursos en línea, mediante aportes que pueden hacer a preguntas generadoras de debates, formuladas por el profesor o por los mismos estudiantes.

Según Barberà (2004), los foros se conforman mediante un proceso de interacción, principalmente entre alumnos, pero también con la participación o moderación del profesor, por medio de intercambios comunicativos escritos dirigidos a discutir y aportar argumentos sobre la temática de debate, con la finalidad de compartirlos y mejorar el aprendizaje de cada uno de los participantes suscritos al foro.

En tal sentido, el papel del profesor en un foro de discusión puede ir desde ser un simple espectador situándose fuera del debate, hasta ser un promotor de las discusiones, integrándose como un participante más, naturalmente, aportando contribuciones esclarecedoras como experto en el tema de discusión. También el profesor puede actuar como un moderador o animador de los debates, con preguntas provocadoras que deben estimular la participación de los estudiantes (Barberà, 2004). En todo caso, en la planificación de foros el diseñador debe fomentar y requerir la participación activa de los estudiantes.

Además, en la organización de un foro virtual, se dan tres fases esenciales: a) enunciado del tema de debate, y delimitación de la normativa que lo regirá (cuantas veces se debe participar, quién, cuando, de qué modo, el papel del profesor, etc.); b) realización de una clarificación de posturas, 
producción de nuevas aportaciones y argumentos, y réplicas que favorezcan la fijación de posiciones sobre determinados puntos del tema elegido; y c) realización de actividades de revisión y síntesis de consensos establecidos de manera explícita para llegar a una conclusión integradora de las diferentes opiniones aportadas (Barberà, 2004).

El mayor reto en la planificación y organización de un foro está en conseguir que los estudiantes participen activamente y que además lean y reflexionen sobre los aportes dados por sus compañeros. Sobre este particular, siguiendo a Cole (2005) y Rice (2006) se recomiendan las siguientes directrices:

1. Haga de los foros una parte importante de sus clases, pero, no sobrecargue el curso con foros en todos los temas o semanas. Maneje la apertura de foros con mucho cuidado, para que esta actividad no se revierta en trabajo excesivo ocasionado por su obligación de leer todas las intervenciones, responder algunas de ellas, y calificarlas. En otras palabras, aunque se les considera una estrategia importante, no se extralimite en la creación de foros.

2. Maneje a tiempo las expectativas de sus estudiantes. Todo estudiante que publica en un foro aspira ser leído pronto, especialmente por el profesor. Deje claro ante sus estudiantes con qué frecuencia usted revisará los mensajes. Por ejemplo, aclare si usted estará chequeando los mensajes diariamente, cada dos días o semanalmente. Si usted no aclara esto, los estudiantes asumirán que usted estará en línea las 24 horas al día y noche, durante toda la semana, especialmente si usted ha creado un FORO para procesar dudas.

3. Deje bien claras desde un principio las reglas sobre su participación y las de los estudiantes en el foro. Por ejemplo, para evitar que los alumnos sólo hagan sus intervenciones originales, exija que por lo menos presenten dos o tres réplicas a los aportes dados por otros compañeros.

4. Estudie la conveniencia de habilitar al menos un foro donde sean los estudiantes quienes asuman el rol protagónico de moderar las discusiones. Subdivídalos por grupos, y que cada grupo se haga responsable de presentar una temática y administrar el debate. En principio, para evitar que se discuta sobre cualquier cosa irrelevante para el curso, usted puede sugerir la temática para cada grupo de acuerdo con los objetivos de aprendizaje propuestos.

5. Tenga presente que los foros de aprendizaje se conforman mediante un proceso de interacción principalmente entre los alumnos. Pero, también con la intervención del profesor, por medio de intercambios comunicativos escritos dirigidos a discutir y aportar argumentos sobre una determinada temática de debate.

6. Tenga en cuenta que un foro de aprendizaje es un espacio de naturaleza estrictamente académica. Por lo tanto, no deben propiciar intervenciones ajenas al logro de los objetivos de aprendizaje. Deben evitarse comentarios que desvíen las discusiones propuestas.

7. Tenga presente que los foros agregan valor a la práctica docente basada en el constructivismo social, en la medida que se haga una adecuada moderación y una apropiada selección de temas que "enganchen" a los estudiantes a debatir asuntos relevantes para el logro de los objetivos de aprendizaje, donde todos aprenden de todos.

8. Estimule la participación de los estudiantes. ¿Cómo? Por ejemplo: Integre las temáticas de los foros a los intereses del curso. Califique las intervenciones. Permita que los alumnos califiquen. Convenza a sus estudiantes sobre la efectividad de estas dos herramientas de aprendizaje: hacer preguntas y dar respuestas. Que los foros se conciben con la finalidad de compartir opiniones e ideas argumentadas para mejorar el aprendizaje de cada uno de los participantes (Barberà, 2004)

9. Sea prudente en sus respuestas a los alumnos, evitando comentarios que pueden hacerse en privado y no en público. Especialmente, evite hacer halagos públicos a los estudiantes sobresalientes por sus aportes en los foros.

10. Sea amable y considerado en sus respuestas, aún en los casos de total discrepancia con la intervención de algún estudiante. Modere los foros con madurez y amplitud de criterio para aceptar posiciones contrarias a las suyas. Con su ejemplo, reafirme valores como: tolerancia, respeto, amabilidad, puntualidad, solidaridad, amistad, honestidad.

El análisis de las respuestas a las preguntas orientadoras de esta fase de la experiencia conjuntamente con los aportes teóricos, condujo a los autores a plantear situaciones que involucraran activamente a los participantes en su propio aprendizaje. Como muestra, para el foro de discusión se diseñaron las siguientes orientaciones:

"Estimados colegas, para cerrar en esta semana con la lectura analítica recomendada sobre los principios pedagógicos de la plataforma Moodle, se les invita a que reflexionen sobre lo siguiente: en el proceso instruccional a distancia el alumno se convierte en el centro de la acción didáctica y según los supuestos del enfoque constructivista del aprendizaje, los estudiantes construyen activamente nuevos conocimientos a medida que interactúan con su entorno (Driscoll, 2000). A la luz de estos supuestos, ¿cómo asumiría usted el rol de docente constructivista en la EaD? En general, ¿cómo caracterizaría usted a un docente constructivista en los entornos virtuales de enseñanza y aprendizaje? Tómese su tiempo, realice las lecturas recomendadas y elabore y revise su intervención y proceda a participar en este espacio. Argumente sus respuestas. Recuerde, si desea que sus compañeros disfruten la lectura de sus comentarios, procure ser breve y conciso. Haga sus aportes originales y por favor, replique por lo menos dos mensajes de sus compañeros, bien mostrando divergencia o acuerdo 
con sus planteamientos. Saludos cordiales, los facilitadores”

Asimismo, se diseñaron las siguientes orientaciones para el ensayo individual: Escriba un ensayo con una extensión máxima de dos páginas tamaño carta, a espacio sencillo, márgenes simétricos de 2,54cms, fijando su posición sobre las siguientes interrogantes ¿¿cuáles aspectos relacionados con el alumno considera usted como difíciles de controlar al planificar evaluaciones de los aprendizajes a distancia? ¿Es factible evaluar a distancia? ¿Cómo resumiría usted la contribución de las tecnologías para la evaluación de los aprendizajes a distancia? Argumente sus respuestas. Se recomienda visitar la siguiente página Web que trata sobre el empleo de las nuevas tecnologías en la
evaluación
de
los aprendizajes
a
distancia

http://www.ateneonline.net/datos/64_03_Romera_Carolina.pdf

Evaluar y Revisar. Esta fase tiene como propósitos: (a) revisar con sentido crítico y constructivo el desarrollo de todo el proceso de instrucción para determinar sus aciertos y desaciertos; (b) determinar en los aprendices su nivel de aprovechamiento o logro de los resultados previstos en los objetivos de aprendizaje o las competencias planteadas; (c) evaluar la instrucción, midiendo la efectividad de sus métodos y recursos; (d) determinar las discrepancias entre los resultados previstos y los logrados al final de la instrucción; (e) determinar las deficiencias de los medios, métodos o materiales usados; y (f) precisar en forma cualitativa el nivel de satisfacción alcanzado, tanto por el facilitador como por los participantes.

Algunas de las preguntas que orientaron a los autores en esta fase del modelo fueron las siguientes:

¿Qué tipo de instrumentos deben ser diseñados y validados para evaluar el curso en cuanto a sus objetivos, materiales, medios y métodos usados?

¿Qué tipo de instrumentos deben ser diseñados y validados para sondear el nivel de satisfacción de los participantes con el desempeño del facilitador?

¿Se atendieron oportunamente los intereses y necesidades de los aprendices?

¿Son satisfactorios los resultados del rendimiento académico de los participantes como evidencia de logro de las competencias propuestas?

¿Las actividades desarrolladas fueron pertinentes para el logro de las competencias y motivadoras para los participantes?

¿Las actividades de evaluación fueron coherentes con los objetivos propuestos y con las actividades de aprendizaje empleadas?
¿Cuál es el nivel de satisfacción de los participantes con el desarrollo del curso?

¿Se presentaron inconvenientes de carácter administrativo o tecnológico para el desarrollo del curso? ¿Cómo se resolvieron?

Con base en el análisis de las respuestas a las preguntas antes planteadas se decidió aplicar una encuesta a los participantes con la finalidad de determinar su nivel de satisfacción y actitudes con respecto a los siguientes aspectos: Publicación oportuna de los materiales de instrucción; puntualidad del facilitador en las actividades de Chat; formulación oportuna y pertinencia de las preguntas generadoras de debate en los foros de discusión; realimentación útil y oportuna del facilitador por vía de correo electrónico; nivel de operatividad de la plataforma RED-UCLA en cuanto a su accesibilidad, rapidez y funcionamiento continuo; utilidad, claridad de exposición y contenido del tutorial; nivel de ayuda o tutoría prestada por el facilitador, contenido programático y nivel del curso.

Se evaluaron las opiniones expresadas en forma anónima por los participantes, y se hicieron los ajustes necesarios para mejorar los aspectos cuya efectividad fue cuestionada. Entre otros, se encontraron los siguientes resultados: el curso fue útil para ayudar a los participantes en la construcción de su propio aprendizaje y facilitó el aprendizaje del modelo ASSURE como formato de planificación instruccional en línea. Los medios y estrategias empleadas realmente ayudaron a mantener una comunicación oportuna y fluida entre los participantes y entre éstos y sus facilitadores. Los debates en línea contribuyeron a desarrollar habilidades para la crítica constructiva, la tolerancia y el respeto por las ideas de los demás, así como, la capacidad de argumentar siempre las ideas expuestas con referentes o soportes teóricos. Las orientaciones de los facilitadores en todas las etapas del proceso instruccional fueron útiles para favorecer la transferencia de cada una de las etapas del modelo ASSURE en la planificación de los cursos en línea de cada uno de los participantes. Por otra parte, fueron realizadas algunas observaciones con relación a la baja velocidad y algunas dificultades de acceso que presentó la plataforma empleada.

\section{CONCLUSIONES}

No obstante que el Modelo ASSURE se concibió para orientar el proceso instruccional apoyado con medios y tecnología en un aula tradicional, esta experiencia demostró que su aplicación es posible y pertinente en un contexto de formación a distancia en línea. Este modelo es fácil de seguir y no necesariamente demanda la intervención de equipos multidisciplinarios de planificadores. Es aplicable para eventos instruccionales en línea que requieran una o muy pocas horas de instrucción.

El seguimiento adecuado de las etapas del modelo, permite que los actores principales del proceso instruccional en línea, facilitadores y aprendices a distancia, dispongan de un plan de acción detallado y bien definido para el análisis, diseño, preparación y evaluación 
de la instrucción. Asimismo, los autores concluyen que el modelo propone condiciones para llevar a cabo un proceso de enseñanza efectiva, que conlleva al logro de aprendizajes significativos por parte de los estudiantes.

Finalmente, se consideró recomendar a los docentes que incursionan en la modalidad de educación en línea, modificar y adaptar sus modelos tradicionales de planificación instruccional para ensayar y adoptar nuevos procedimientos y metodologías que contribuyan a mejorar la calidad de los procesos de enseñanza y aprendizaje a distancia; teniendo como base que el proceso de planificación y organización de cursos a distancia debe ocurrir con mucha anticipación al inicio previsto de la instrucción (Simonson, Smaldino, Albright, y Zvacek; 2003). Esto ayudará al docente a eliminar planificaciones orientadas por ensayos y errores que pudieran poner en riesgo el aprendizaje de los estudiantes.

\section{REFERENCIAS}

Ausubel, D., Novak, J., y Henesian, H. (2000). Psicología educativa: un punto de vista cognoscitivo. México, D. F.: Trillas.

Barberà, E. (2004). La educación en la red: actividades virtuales de enseñanza y aprendizaje. Barcelona, España: Paidós.

Cole, J- (2005). Using moodle. Sebastopol, CA: O`Reilly Media.

Cookson, P. (2003). Elementos de diseño instruccional para el aprendizaje significativo en la educación a distancia. [Documento en línea] Disponible: http://meidi.ula.ve/ dinstruccional/archivos/elementos.doc [Consulta: 2006, Noviembre 10].

Díaz-Barriga, F. y Hernández, G. (2002). Estrategias docentes para un aprendizaje significativo: una interpretación constructivista. México, D. F.: McGraw-Hill.

Dick, W., Carey, L., y Carey, J. (2001). The systematic design of instruction. New York: Addison - Wesley Educational Publisher.

Driscoll, M. (2000). Psychology of learning for instruction. Needham Heights, MA: Allyn and Bacon.

Fainholc, B. (1999). La interactividad en la educación a distancia. Buenos Aires: Paidos.

Gagné, R. y Briggs, L. (2001). Planificación de la enseñanza: sus principios. México, D.

\section{F.: Trillas.}

Gronlund, N. (2000). How to write and use instructional objectives. Upper Saddle River, NJ: Prentice-Hall.

Gustafson, K. y Branch, R. (2002). Survey of instructional developments models. Syracuse, NY: ERIC Clearinghouse on Information \& Technology.

Heinich, R., Molenda, M., Russell, J., y Smaldino, S. (2002). Instructional media and technology learning. Upper Saddle River, NJ: Pearson Education.

Hernández, M. (2006). Introducción a Moodle. [Documento en línea] Disponible: http://ead. ucla.edu.ve/course/view.php?id=20 [Consulta: 2006, Noviembre 10]

Pelz, B. (2004). (My) three principles of effective online pedagogy. [Documento en línea] Disponible: http://www.aln.org/publications/jaln/v8n3/pdf/v8n3_pelz.pdf [Consulta: 2006, Marzo 15].

Rice, W. (2006). Moodle: E.learning course development. Birmingham, UK: Packt Pub.

Schlosser, L. y Simonson, M. (2002). Distance education; Definition and glossary of terms. Bloomington, IN: AECT.

Simonson, M., Smaldino, S., Albright, M., y Zvacek, S., (2003) Teaching and Learning at a Distance: Foundations of Distance Education. New Jersey: Merrill Prentice Hall.

Smith, P. y Ragan, T. (1999). Instructional design. Hoboken, NJ: John Wiley and Sons.

Tobón, S. (2006). Formación basada en competencias: Pensamiento complejo, diseño curricular y didáctica. Bogotá, Colombia: Ecoe Ediciones. 